Article

\title{
Photodynamic Opening of the Blood-Brain Barrier Using Different Photosensitizers in Mice
}

\author{
Oxana Semyachkina-Glushkovskaya ${ }^{1, *}$, Ekaterina Borisova ${ }^{1,2, *}$, Vanya Mantareva ${ }^{3}$, \\ Ivan Angelov ${ }^{3}$, Ivelina Eneva ${ }^{3}$, Andrey Terskov ${ }^{1}$, Aysel Mamedova ${ }^{1}$, Alexander Shirokov 1,4,5, \\ Alexander Khorovodov ${ }^{1}$, Maria Klimova ${ }^{1}$, Ilana Agranovich ${ }^{1}$, Inna Blokhina ${ }^{1}$ (D), \\ Nikita Lezhnev ${ }^{1}$ and Jurgen Kurths ${ }^{1,6,7}$
}

1 Saratov National Research State University, 83, Astrakhanskaya str., 410012 Saratov, Russia; terskow.andrey@gmail.com (A.T.); mamedovayse195@gmail.com (A.M.); shirokov_a@ibppm.ru (A.S.); khorovodov2012@yandex.ru (A.K.); mari-1997@mail.ru (M.K.); ilana.agranovich@yandex.ru (I.A.); inna-474@yandex.ru (I.B.); nik.lezhnev.2018@mail.ru (N.L.); kurths@pik-potsdam.de (J.K.)

2 Institute of Electronics, Bulgarian Academy of Sciences, 72, Tsarigradsko Chaussee Blvd., 1784 Sofia, Bulgaria

3 Institute of Organic Chemistry with Centre of Phytochemistry, Bulgarian Academy of Sciences, Acad. G. Bonchev, str., Bld. 9, 1113 Sofia, Bulgaria; mantareva@yahoo.com (V.M.); ipangelov@gmail.com (I.A.); ivelina.eneva6@gmail.com (I.E.)

4 Institute of Biochemistry and Physiology of Plants and Microorganisms, Russian Academy of Sciences, Prospekt Entuziastov 13, 410049 Saratov, Russia

5 Saratov State Medical University, Bolshaya Kazachia st., 112, 410012 Saratov, Russia

6 Physics Department, Humboldt University, Newtonstrasse 15, 12489 Berlin, Germany

7 Potsdam Institute for Climate Impact Research, Telegrafenberg A31, 14473 Potsdam, Germany

* Correspondence: glushkovskaya@mail.ru (O.S.-G.); ekaterina.borisova@gmail.com (E.B.); Tel.: +7-927-115-5157 (O.S.-G.); +359-879-497-118 (E.B.)

Received: 5 November 2019; Accepted: 17 December 2019; Published: 19 December 2019

\begin{abstract}
In a series of previous studies, we demonstrated that the photodynamic therapy (PDT), as a widely used tool for treatment of glioblastoma multiforme (GBM), also site-specifically opens the blood-brain barrier (BBB) in PDT-dose and age-related manner via reversible disorganization of the tight junction machinery. To develop the effective protocol of PDT-opening of the BBB, here we answer the question of what kind of photosensitizer (PS) is the most effective for the BBB opening. We studied the PDT-opening of the BBB in healthy mice using commercial photosensitizers (PSs) such as 5-aminolevulenic acid (5-ALA), aluminum phthalocyanine disulfonate (AlPcS), zinc phthalocyanine $(\mathrm{ZnPc})$ and new synthetized PSs such as galactose functionalized $\mathrm{ZnPc}(\mathrm{GalZnPc})$. The spectrofluorimetric assay of Evans Blue albumin complex (EBAC) leakage and 3-D confocal imaging of FITC-dextran $70 \mathrm{kDa}$ (FITCD) extravasation clearly shows a revisable and dose depended PDT-opening of the BBB to EBAC and FITCD associated with a decrease in presence of tight junction (TJ) in the vascular endothelium. The PDT effects on the BBB permeability, TJ expression and the fluorescent signal from the brain tissues are more pronounced in PDT-GalZnPc vs. PDT-5-ALA/AlPcS/ZnPc. These pre-clinical data are the first important informative platform for an optimization of the PDT protocol in the light of new knowledge about PDT-opening of the BBB for drug brain delivery and for the therapy of brain diseases.
\end{abstract}

Keywords: blood-brain barrier; photodynamic effects; photosensitizers; drug brain delivery

\section{Introduction}

The blood-brain barrier (BBB) is a major hindrance for the effective delivery of therapeutic compounds to the brain [1]. Various strategies to overcome the BBB have been investigated, including 
direct intranasal drug delivery [2], focused ultrasound [3], intra-arterial infusion of mannitol [4], or direct intracranial infusion [5]. Nowadays the photodynamic effect on the BBB is actively discussed as a new strategy for drug brain delivery, especially for post-surgery therapy of glioblastoma multiforme (GBM) [6]. Photodynamic therapy (PDT) is a widely used tool for therapy of GBM [7,8]. The PDT is based on a laser excitation of photosensitizers (porphyrins, chlorins, and many other photosensitive dyes) that is accompanied by producing singlet oxygen $\left({ }^{1} \mathrm{O}_{2}\right)$ which oxidizes biomolecules causing cancer cell apoptosis and/or necrosis through plasma and mitochondrial membrane rupture. Recently, it has been revealed that PDT can also effectively and site-specifically open the BBB [9-14]. For the first time a targeted disruption of the BBB following a 5-aminolevulenic acid (5-ALA) mediated PDT via the opened skull was shown by Hirschberg et al. in 2008 [9]. Later Madsen et al. demonstrated a non-invasive PDT-opening of the BBB for macrophages using a $670 \mathrm{~nm}$ laser and aluminum phthalocyanine disulfonate (AlPcS) [10]. In a series of our experimental studies of PDT-5-ALA-opening of the BBB we have analyzed the optimal dose of $635 \mathrm{~nm}$ irradiation and 5-ALA, the time window for PDT-opening/closing of the BBB, mechanisms underlying this phenomenon and age differences in PDT-related increase in the BBB permeability, which is consistent with other data [11-15]. In this work we attempt to answer the question of what kind of photosensitizer might be most effective for PDT-opening of the BBB. Therefore, we analyzed the BBB permeability to high molecular weight molecules using two types of photosensitizers (PS) such as 5-ALA and phthalocyanines (PCs) since their application for drug delivery systems has been shown in other studies $[16,17]$.

Phthalocyanines (PCs) are synthetic dyes that were obtained and described already in 1907 by Braun and Tcherniac [18]. PCs consist of four units of isoindoles bridged by nitrogen atoms and which form one aromatic heterocyclic structure. Main photophysical characteristics of PCs are: absorption spectrum with two important bands- the weak Soret-band located in the UV zone of the spectrum with maximum at 350-360 nm and the Q-band in the red zone of the visible spectrum at around $670-680 \mathrm{~nm}$. The high intensity of the latter band, due to values of the molar extinction coefficient $1 \times 10^{5} \mathrm{M}^{-1} \mathrm{~cm}^{-1}$ (two orders of magnitude higher than that at most of porphyrins) is one of the main characteristics of these dyes [19]. Photochemical properties of PCs are strongly influenced by the nature of the coordinated central metal ion. Thus, the presence of diamagnetic elements in the central cavity, such as zinc (II), aluminum (III), gallium (III) or silicon, gives them a high triplet quantum yield $\left(\Phi_{\mathrm{T}}>0.4\right)$ with long triplet lifetimes $\left(\tau_{\mathrm{T}}>100 \mu \mathrm{s}\right)$. Triplet energy states with energy of about $1.2 \mathrm{eV}$, make them efficient generators of singlet oxygen with values of singlet oxygen quantum yields (SOQY) more than $0.4[20,21]$.

We hypothesize that functionalization of PCs by galactose might be more effective for the PDT-mediated BBB opening due to enhancing the trans-endothelial permeability by galactose via activation of protein kinase C (PKC), a family of kinases vital to intracellular signaling that is a common effect of monosaccharides on vascular endothelium [22,23]. Our previous results indicate that functionalization of PCs by galactose is more favorable than zinc only due to their chemical properties [24,25]. Based on these facts, here we study the BBB opening in healthy mice using the PDT protocol with different PS, including commercial PS such as 5-ALA and AlPcS as well as new synthesized PS such as zinc phthalocyanine ( $\mathrm{ZnPc}$ ) and functionalized by galactose $\mathrm{ZnPc}(\mathrm{GalZnPc})$.

\section{Materials and Methods}

\subsection{Subject}

Experiments were performed in mongrel male mice $(25 \mathrm{~g})$ in accordance with the Guide for the Care and Use of Laboratory Animals published by the U.S. National Institutes of Health (NIH Publication No. 85-23, revised 1996), protocols were approved by the Institutional Review Board of the Saratov State University (Protocol 7, 07.02.2017). The rats were housed at $25 \pm 2{ }^{\circ} \mathrm{C}, 55 \%$ humidity, and $12 \mathrm{~h}: 12 \mathrm{~h}$ light-dark cycle. Food and water were given ad libitum. 
The experiments were performed in the following groups: (1) three control groups, including mice without PDT (1a) the intact mice, (1b) no laser, (1c) no PS, injection of physiological saline (sham group); (2) four PDT groups, including mice after PDT using different PSs (2a) 5-ALA; (2b) AlPcS; (2c) $\mathrm{ZnPc}$; (2d) GalZnPc; $\mathrm{n}=10$ in each group in all series of experiments.

\subsection{Photodynamic Treatment Protocol}

The mice were anaesthetized with ketamine (100 mg/kg, i.p.) and xylazine (10 mg/kg, i.p.)., and fixed in a stereotactic frame. The PDT was performed $30 \mathrm{~min}$ after intravenous injection of PS (5-ALA, ALASENS, Niopik Inc., Moscow, Russian Federation, $20 \mathrm{mg} / \mathrm{kg}$ ); AlPcS, ZnPc and the galactosilated zinc phtalocianine (GalZnPc) were synthesized according to procedures described in our previous papers $[18,19,26,27]$ with modifications depending on coordinating metal. Photodynamic treatment protocol is presented on Scheme 1.

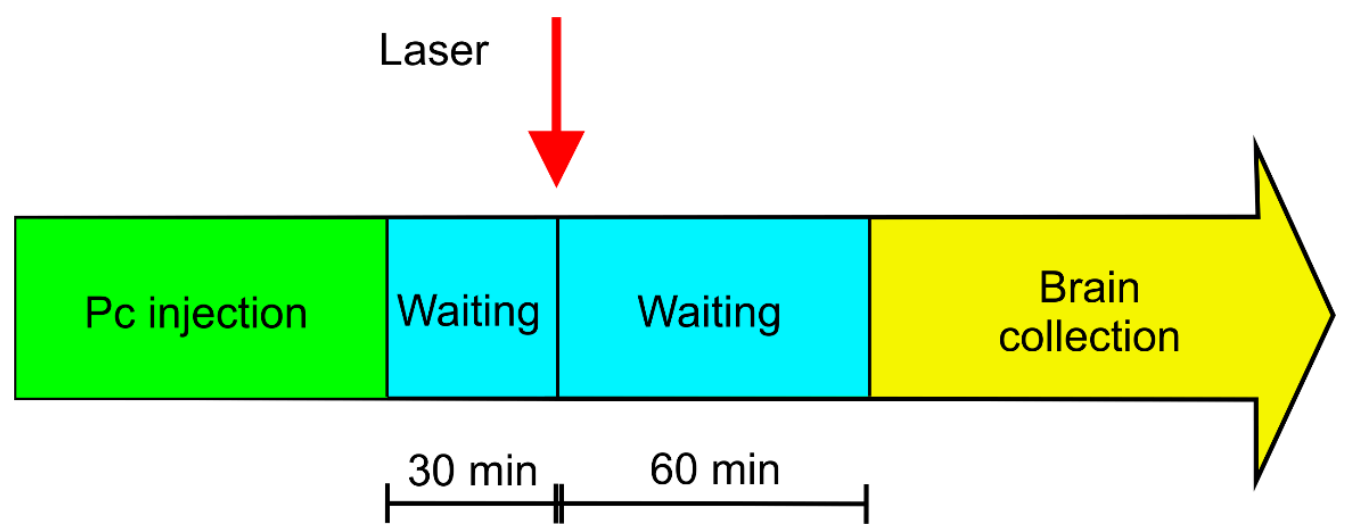

Scheme 1. Illustration of the time points for phthalocyanine injection and laser irradiation in relation to photodynamic treatment-mediated opening of the BBB and the brain collection.

The epicranium was exposed by a parieto-occipital midline skin incision. With the use of a microsurgical technique, the periosteum was pushed back, and biparietal parasagittal groove-shaped trephination $(2 \times 2 \mathrm{~mm})$ was performed with a microdrill (Craniotome Drill, Aesculap Inc., Pennsylvania, USA) accompanied by a continuous irrigation with saline to prevent heating of the tissue. Special care was taken to avoid a penetration of the dura mater.

A preliminary quantitative evaluation of the photosensitizer appearance in brain tissue was made using fluorescence detection in the red and near-infrared region (600-1000 nm). Excitation was used in the Soret-band of the sensitizers, and the light source applied was LED with maximum of emission at $400 \mathrm{~nm}$ and full width half maximum - FWHM $=20 \mathrm{~nm}, 25 \mathrm{~mW}$ output power (LED405, Polironik Ltd., Moscow, Russia). Fiberoptic probe $6+1$ was used for delivery of excitation and emission light from the brain tissues. Microspectrometer USB4000 (OceanOptics Inc., Dunedin, USA) was used for detection of the exogenous fluorescence signals.

For irradiation at $635 \mathrm{~nm}$, we used a high-power continuous-wave red LED (XPeBRD-L1-0000-00901, CREE, Inc.) emitting up to $1 \mathrm{~W}$ output light power with maximum at $635 \mathrm{~nm}$, with a possibility to control the emitted-light power density in the range of $20-200 \mathrm{~mW} / \mathrm{cm}^{2}$ at a step of $10 \mathrm{~mW} / \mathrm{cm}^{2}$, as measured on the tip surface of an 8-mm diameter solid light-guide. The spectral width of the emitted light at full width at half maximum (FWHM) of intensity was $30 \mathrm{~nm}$. For irradiation at $670 \mathrm{~nm}$ we used a laser diode (RLT67300T, Roithner, Vienna, Austria) with $300 \mathrm{~mW}$ maximum output power.

The murine brains sensitized with phthalocyanine compounds were also irradiated with a constant power density of $40 \mathrm{~mW} / \mathrm{cm}^{2}$ and exposure times of $62.5 \mathrm{~s}, 125 \mathrm{~s}$, and $250 \mathrm{~s}$ to achieve a light dose of $2.5 \mathrm{~J} / \mathrm{cm}^{2}, 5 \mathrm{~J} / \mathrm{cm}^{2}$ and $10 \mathrm{~J} / \mathrm{cm}^{2}$, respectively, for all types of photosensitive drugs applied. 
The heating of the brain tissue caused by exposure to light was monitored by using a thermocouple data logger (Pico Technology, USB TC-08, Cambridgeshire, UK).

No significant temperature increase was observed during the light irradiation of the brain tissues. The temperature rise did not exceed $2{ }^{\circ} \mathrm{C}$ above the mouse body level of $28^{\circ} \mathrm{C}$ for the irradiation doses chosen.

\subsection{Spectrofluorometric Assay of Evans Blue Albumin Complex (EBAC) Extravasation}

Three days before the experiments, the polyethylene catheter was implanted into the femoral vein for intravenous injection of Evans blue dye in awake mice. The implantation of a catheter was performed under anesthesia with ketamine $(100 \mathrm{mg} / \mathrm{kg}$, i.p. $)$ and xylazine $(10 \mathrm{mg} / \mathrm{kg}$, i.p.). Before PDT Evans blue dye was injected and circulated in the blood for $30 \mathrm{~min}$ in accordance with our protocol $[11,12]$. At the end of the circulation time, mice were decapitated; their brains and blood were quickly collected and placed on ice (no anti-coagulation was used during blood collection). Prior to brain removal, the brain was perfused with saline to wash out the remaining dye in the cerebral vessels.

The detailed protocol of Evans blue albumin complex (EBAC) extraction and visualization was published by Wang et al. [28]. Briefly, the isolated brains were cut into small pieces and incubated with saline (1:3) for $60 \mathrm{~min}$ to enable the soluble substances to dissolve. Then the solutions were centrifuged at $10,000 \times g$ for $10 \mathrm{~min}$ to sediment the non-dissolved parts. The supernatants that contained the brain solutes were treated by $50 \%$ trichloroacetic acid-TCA (1:3), centrifuged again at 10,000 $\mathrm{g}$ for $20 \mathrm{~min}$ to remove precipitated molecules. The final supernatants were incubated in $95 \%$ ethanol to increase the optical signals for the spectrofluorometric assay $(620 \mathrm{~nm} / 680 \mathrm{~nm}$, Agilent Cary Eclipse, Agilent, USA) on the base of the CCU "Symbiosis" IBPPM RAS. The standard calibration curve was created and used for calculation of the EBAC concentration ( $\mu$ g per $g$ of tissues).

Despite the fact that a quantitative analysis of EBAC is still the most commonly used marker of the BBB integrity, there are some problems arising from its ex vivo and in vivo application: about $15 \%$ of Evans blue dye remains on the endothelium of cerebral vessels; Evans blue dye can be taken by red blood cells and astrocytes [29]. Therefore, for qualitative and quantitative assessment of the BBB integrity, we used additional methods for the analysis of the BBB permeability to low and high molecular weight molecules.

\subsection{Confocal Microscopy of FITC-dextran $70 \mathrm{kDa}$ Extravasation}

Fluorescein isothiocyanate (FITC)-dextran $70 \mathrm{kDa}$ (FTICD) was used as an additional valuable tool to characterize the BBB permeability to high molecular weight molecules. The protocol for FITCD to assess the BBB disruption using confocal microscopy is described in detail in [30]. Briefly, before (in the intact mice) or $1 \mathrm{~h} / 4 \mathrm{~h} / 24 \mathrm{~h}$ after PDT, FITCD $70 \mathrm{kDa}(1 \mathrm{mg} / 25 \mathrm{~g}$ mouse, $0.5 \%$ solution in saline, Sigma-Aldrich) was injected into the tail vein and allowed to circulate for $2 \mathrm{~min}$. Afterward mice were decapitated and the brains were quickly removed and fixed in $4 \%$ paraformaldehyde for $24 \mathrm{~h}$, cut into 50- $\mu \mathrm{m}$ thick slices on a vibratome (Leica VT 1000S Microsystem, Germany) and analyzed using a confocal microscope (Olympus FV10i-W, Olympus, Japan). Approximately 8-12 slices per animal from cortical and subcortical (excepting hypothalamus and choroid plexus where the BBB is leaky) regions were imaged. The pericytes marker NG2 (1:500, ab 50009, Abcam, Cambridge, UK) was used for labeling of the cerebral vessels using the protocol for immunolabelling assay.

\subsection{Immunohistochemical Assay}

Mice were euthanized with an intraperitoneal injection of a lethal dose of ketamine and xylazine and intracardially perfused with $0.1 \mathrm{M}$ of phosphate-buffered saline for $5 \mathrm{~min}$. Afterward, the brains were removed and fixed in $4 \%$ buffered paraformaldehyde for one day and in $20 \%$ sucrose for another day. Fluorescence of antigens on free-floating sections was evaluated using the standard method of simultaneously combined staining (Abcam Protocol). Brain slices $(50 \mu \mathrm{m})$ were blocked in $150 \mu \mathrm{L} \mathrm{10 \%}$ bovine serum albumin/ $0.2 \%$ Triton X-100/PBS for $2 \mathrm{~h}$, then incubated overnight at $4{ }^{\circ} \mathrm{C}$ and $2 \mathrm{~h}$ at room 
temperature with Clauding-5 (CLND-5, 1:500; Santa Cruz Biotechnology, sc-28670, Santa Cruz, USA); Occludin (Occ, 1:500; Abcam, ab58803, Cambridge, USA); junctional adhesion molecule (JAM, 1:500, Abcam, ab1316, Cambridge, USA). After several rinses in PBS, the slides were incubated for $3 \mathrm{~h}$ at room temperature with fluorescent-labeled secondary antibodies on $1 \%$ BSA $/ 0.2 \%$ Triton X-100/PBS (1:500; Goat A/Rb, Alexa 555- Abcam, UK, ab150078). Confocal microscopy of the mice cerebral cortex was performed using a fully automated confocal laser scanning microscope with water immersion Olympus FV10i-W (Olympus, Japan). The ImageJ software was used for image data processing and analysis. The areas of fluorescence from TJ were calculated using the plugin "Analyze Particles" in the Analyze tab, which calculates the total area of antigen-fluorescence-the indicator "Total Area." In all cases, 10 regions of interest were analyzed.

\subsection{Statistical Analysis}

The results were reported as a mean value \pm standard error of the mean (SEM). Differences from the initial level in the same group were evaluated by the Wilcoxon test. Intergroup differences were evaluated using the Mann-Whitney test and the ANOVA-2 (post hoc analysis with the Duncan's rank test). The significance levels were set at $p<0.05$ for all analyses.

\section{Results}

\subsection{Phthalocyanine Functionalization by Galactose}

The synthetic procedure for the galactosilated phthalocyanine-galactopyranosyl Zn(II)Pc—is according to the presented synthetic scheme (Scheme 2) and partly described in [31,32].
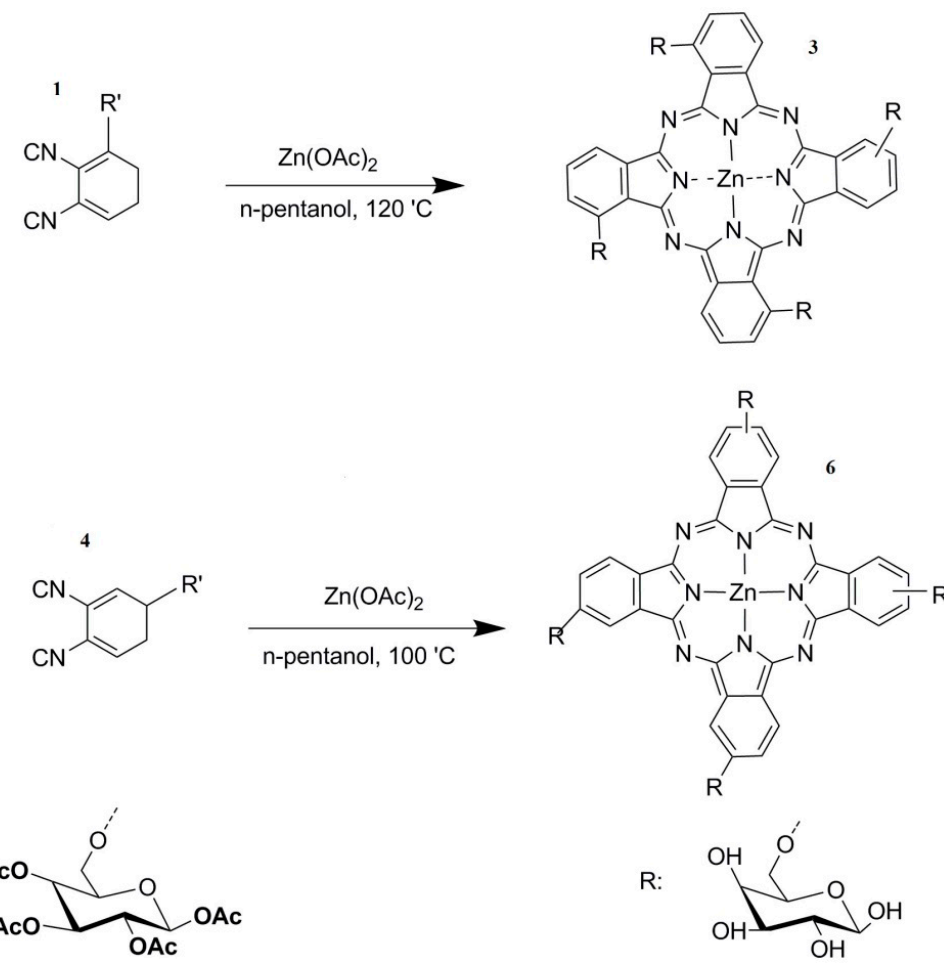

Scheme 2. Sequence of synthetic procedures for the synthesis of tetra-substituted $\mathrm{Zn}$ (II) phthalocyanine with $\alpha$-D-galactopyranose as functional group in peripheral and non-peripheral positions.

In short, the synthesis procedure involves the following steps: 3-nitrophthalonitrile and 4-nitrophthalonitrile are suitable starting compounds for the synthesis of various non-peripheral and peripherally substituted phthalocyanines. The synthesis of the starting phthalonitrile-substituted galactopyranosyl groups (1) and (4) - precursors in the synthesis of phthalocyanines-proceeds via the 
mechanism of nucleophilic replacement of the $\mathrm{NO}_{2}$ group in DMF solution in the presence of a potassium carbonate base. A further step of the synthesis procedure involves the preparation of tetra-substituted conjugates of $\mathrm{Zn}(\mathrm{II})$ phthalocyanine with protected galactopyranose in a non-peripheral and a peripheral position. The reaction is cyclotetramerization of the resulting substituted phthalonitriles, which is run in a n-pentanol solution and in the presence of a catalyst: 1,8-diazabicyclo[5.4.0]undec-7-ene (DBU) under a nitrogen atmosphere.

The final step of the synthesis procedure consists in unblocking the protective isopropylidene groups of the bound $\alpha$-D-galactopyranose to $\mathrm{ZnPc}$ in a non-peripheral and a peripheral position (respectively compounds 3 and 6). During the investigations, tetra-substituted $\mathrm{Zn}$ (II) phthalocyanine conjugates with protected galactopyranose in a non-peripheral and a peripheral position were used as final products: [n-GalZnPc, p-GalZnPc]. The compounds were chemically characterized and their main absorption and fluorescence properties and the formation of singlet oxygen in their presence were investigated.

\subsection{Photodynamic Opening of the BBB Using Different PSs}

In this session of experiments, we studied the PDT-opening of the BBB using different PSs, including two commercial PSs such as 5-ALA and AlPcS as well as new synthetized ones, such as ZnPc and its functionalized form GalZnPc.

Figure 1-I illustrates molecular formulas of photosensitizers used for PDT-opening of the BBB. Figure 1-II,III demonstrate confocal imaging of the BBB permeability to FITCD before and after PDT. In the control groups (no PDT, no laser or PSs), FITCD was observed only in the cerebral vessels (Figure 1-I). PDT-5-ALA/AlPcS/ZnPc/GalZnPc induced strong FITCD extravasation from the cerebral vessels into the brain tissues. The FITCD leakage was visualized by fluorescence outside the vessel walls (Figure 1-II).

Spectrofluorimetric assay revealed laser dose-mediated increase in the BBB permeability to EBAC $1 \mathrm{~h}$ after PDT-5-ALA/AlPcS/ZnPc/GalZnPc. Figure 1-IV illustrates more pronounced effects of PDT-ZnPc-Gal on the EBAC leakage compared with other types of PSs (GalZnPc/AlPcS: $29.54 \pm 0.02$ vs. $18.64 \pm 0.03, p<0.001$ for $2.5 \mathrm{~J} / \mathrm{cm}^{2} ; 31.80 \pm 0.03$ vs. $20.37 \pm 0.08, p<0.001$ for $5 \mathrm{~J} / \mathrm{cm}^{2} ; 33.93 \pm 0.05$ vs. $23.18 \pm 0.07, p<0.001$ for $10 \mathrm{~J} / \mathrm{cm}^{2}$; GalZnPc/ZnPc: $29.54 \pm 0.02$ vs. $12.55 \pm 0.05, p<0.001$ for $2.5 \mathrm{~J} / \mathrm{cm}^{2} ; 31.80 \pm 0.03$ vs. $14.12 \pm 0.01, p<0.001$ for $5 \mathrm{~J} / \mathrm{cm}^{2} ; 33.93 \pm 0.05$ vs. $15.44 \pm 0.05, p<0.001$ for $10 \mathrm{~J} / \mathrm{cm}^{2}$; GalZnPc/5-ALA: $29.54 \pm 0.02$ vs. $1.18 \pm 0.02, p<0.001$ for $2.5 \mathrm{~J} / \mathrm{cm}^{2} ; 31.80 \pm 0.03$ vs. $1.09 \pm 0.02$, $p<0.001$ for $5 \mathrm{~J} / \mathrm{cm}^{2} ; 33.93 \pm 0.05$ vs. $8.97 \pm 0.05, p<0.001$ for $\left.10 \mathrm{~J} / \mathrm{cm}^{2}\right)$. The level of EBAC in the brain tissues after PDT-GalZnPc/AlPcS/ZnPc/5-ALA was 89-62-48-22-fold higher compared with the control groups (average value for three control groups: the intact mice, no laser, no PSs), i.e., the effects of PDT-GalZnPc on the BBB were 1.4-1.8-4.0 greater than PDT-AlPc1/ZnPc/5-ALA, respectively. Thus, our results clearly demonstrate that all PSs are effective for PDT-mediated opening of the BBB. However, functionalized GalZnPc gives even a stronger increase in the BBB permeability than 5-ALA, AlPcS, and $\mathrm{ZnPc}$, probably due to enhancing the transendothelial permeability by galactose $[33,34]$.

No leakage of FITCD or EBAC was observed $4 \mathrm{~h}$ and $24 \mathrm{~h}$ after PDT, i.e., PDT-opening of the BBB is associated with a rapid recovery of the BBB integrity.

The PDT-opening of the BBB independent of the type of PSs was accompanied by a similar decrease in fluorescence of TJ proteins such as CLND, OCC, and JAM that can explain an increase in the BBB permeability to EBAC and FITCD in mice $1 \mathrm{~h}$ after PDT. It is important to note that the TJ fluorescence was over the normal value $4 \mathrm{~h}$ after PDT suggesting reversibility of PDT-mediated changes in the BBB leakage.

The excitation and emission spectra of the investigated phthalocyanines are quite similar (see Figure 2e-g), with the maxima of excitation and fluorescence respectively at $684 \mathrm{~nm}$ and $691 \mathrm{~nm}$ for AlPcS, $675 \mathrm{~nm}$ and $680 \mathrm{~nm}$ for ZnPc, and 703 and $710 \mathrm{~nm}$ for GalZnPc in polar solution. In biological tissues the maxima of emission are shifted by about $10-12 \mathrm{~nm}$ to a lower wavelength region due to the influence of the microenvironment on the photosensitizer molecules. 

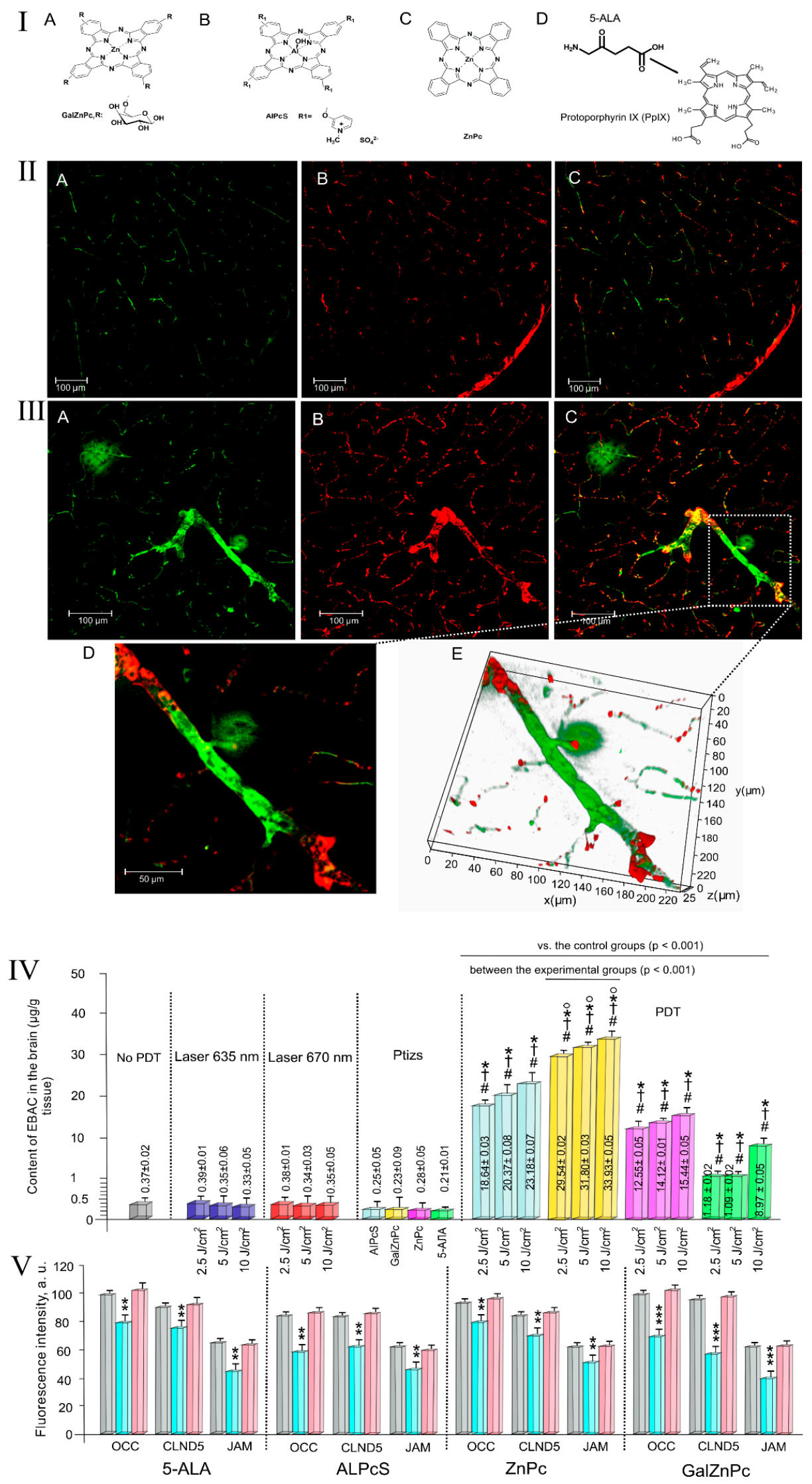

पl before PDT $1 \mathrm{~h}$ after PDT $4 \mathrm{~h}$ after PDT

Figure 1. Photodynamic opening of the BBB using different PSs: 
(I) The chemical formulas of the used PSs (A-GalZnPc; B-AlPcS; C-ZnPc; D-5-ALA) for the BBB opening; (II) The confocal imaging of cerebral vessels (red color) filled by FITCD (green color) in the normal state: A-the cerebral vessels are filled by FITCD (green color), B-the blood vessels marked by NG2 (pericytes marker), C-merged A and B; (III) The confocal imaging of extravasation of FITCD (green color, arrowed) from cerebral vessels (red color) into the brain tissues: A - the cerebral vessels are filled by FITCD (green color), B-the blood vessels marked by NG2 (pericytes marker), C-merged A and B, D and E-2-D and 3-D imaging of FITCD leakage; (IV) Spectrofluorimetric assay of EBAC level in the brain tissues after PDT-opening of the BBB using different PSs (5-ALA/AlPcS/ZnPc/GalZnPc): *; $+; \#-p<0.001$ vs. the control groups including the intact mice, mice after laser irradiation, mice treated by PSs, respectively; $n=10$ for each group and ${ }^{\circ}-p<0.001$ between the experimental groups, at least 10 individual tissue sections taken for each animal for II and III; (V) The fluorescence of TJ proteins in the control group (the intact mice), $1 \mathrm{~h}$ and $4 \mathrm{~h}$ after PDT-5-ALA/AlPcS/ZnPc/GalZnPc $(n=10$ for each group): ${ }^{* *}-p<0.01,{ }^{* * *}-p<0.001$ vs. the control group (the intact mice).

\subsection{Fluorescence Spectra of Brain Tissues with Different PSs}

In these studies, we evaluated the fluorescence signal from exogenous photosensitizers accumulated in brain tissues after opening of BBB. The excitation was used on $400 \mathrm{~nm}$ for all photosensitive drugs, using their Soret-band of absorption. The brain tissue fluorescence of a control group of three mice without exposure to exogenous photosensitive drugs was compared to that of photosensitized animals.

Initially, before red light irradiance of the brain, 5-ALA/PpIX fluorescence was pronounced in a higher degree, in comparison with that of one of phthalocyanine compounds. Moreover $60 \mathrm{~min}$ after irradiation with $635 \mathrm{~nm}$ due to opening of BBB barrier, an increase of this emission was observed in all animals treated, see Figure 2a,b. Some increase of the photosensitizer concentration into brain tissues was observed as well for different phthalocyanines used, namely AlPcS, ZnPc, and GalZnPc after irradiation at $670 \mathrm{~nm}$, but in general the fluorescent intensity observed in the case of phthalocyanine accumulation in brain tissues is about 3 times lower than the emission observed from protoporphyrin IX. The results, obtained with GalZnPc treated animals, are presented in Figure 2c,d. 

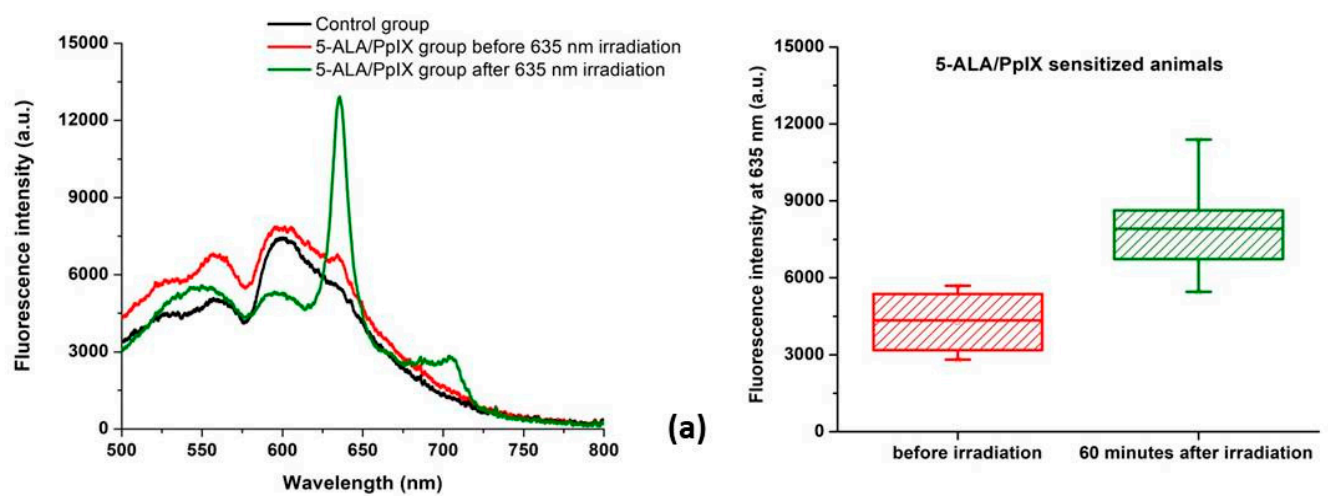

(b)
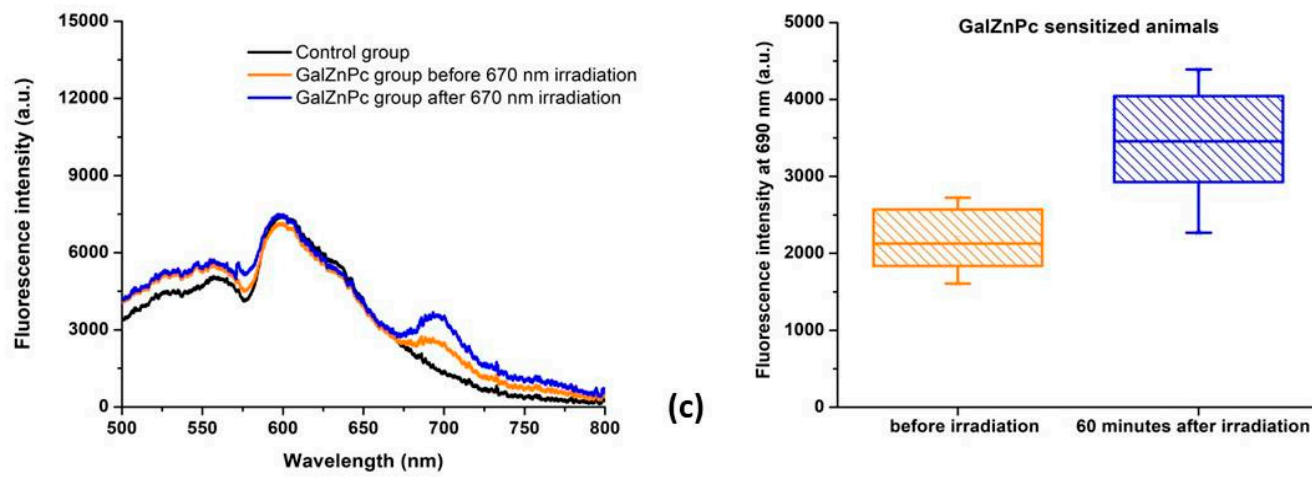

(d)
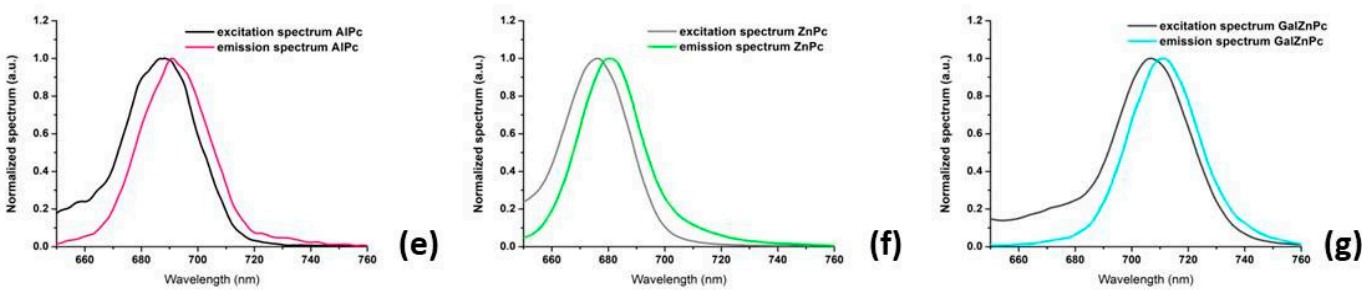

Figure 2. (a) Fluorescence spectra of murine brain in control (untreated with photosensitizer) and experimental group treated with 5-ALA; (b) Fluorescence intensities comparison before and after irradiation with $635 \mathrm{~nm}$ for 5-ALA/PpIX signal obtained from 5-ALA treated mice; (c) Fluorescence spectra of murine brain in control (untreated with photosensitizer) and experimental group treated with GalZnPc; (d) Fluorescence intensities comparison before and after irradiation with $670 \mathrm{~nm}$ for GalZnPc signal obtained from phthalocyanine-treated mice; (e) excitation and emission spectra of $\mathrm{AlPc}$; (f) excitation and emission spectra of $\mathrm{ZnPc}$; (g) excitation and emission spectra of GalZnPc; Spectra in e, $\mathrm{f}$ and $\mathrm{g}$ are normalized with respect to maximum of excitation and emission signals for corresponding phthalocyanines.

\section{Discussion}

In this pre-clinical work on healthy mice, we aimed to answer the question of what kind of photosensitizer is more appropriate for PDT-opening of the BBB. To answer this question, we studied PDT-opening of the BBB using commercial PS such as 5-ALA and AlPcS as well as new synthetized PS such as $\mathrm{ZnPc}$ and functionalized its form GalZnPc. Our results reveal a dose-depended PDT opening of the BBB using all types of PS. The PDT-opening of the BBB using 5-ALA and AlPcS also have been shown by Hirschberg et al. [9], Madsen [10], and in our previous experiments [11-14]. Using spectrofluorimetric assay for quantitative analysis of the BBB permeability to EBAC and confocal imaging of FITCD extravasation, our results demonstrate that PCs induce stronger ECAB leakage compared with 5-ALA. Among PCs, GalZnPc causes the more pronounced increase in the BBB permeability to EBAC vs. AlPcS, ZnPc. Phthalocyanines generate singlet oxygen directly under light irradiation [35], while 5-ALA does not produce single oxygen itself but only via metabolization in the 
cells to protoporphyrin IX [36]. These facts can explain the lesser PDT-5-ALA opening of the BBB to EBAC compared with different forms of used phthalocyanines (AlPc $\backslash \mathrm{S}, \mathrm{ZnPc}, \mathrm{GalZnPc}$ ). The stronger effect on the BBB permeability by GalZnPc can be due to enhancing the transendothelial permeability by galactose via activation of protein kinase $\mathrm{C}$ (PKC), a family of kinases vital to intracellular signaling that is a common effect of monosaccharides on vascular endothelium [22,23].

To better understand mechanisms responsible for PDT-induced opening of the BBB to high molecular weight molecules (EBAC and FITCD), we explored PDT-related changes in the fluorescent signal of TJ trans-membrane proteins such as CLND, OCC, and JAM. The fluorescence of these molecular factors was analyzed before PDT (the control groups), $1 \mathrm{~h}$ after PDT (when BBB was opened), and $4 \mathrm{~h}$ after PDT (when the BBB was closed and completely recovered). Our results uncovered a decrease in the fluorescence of TJ proteins in the time of PDT-opening of the BBB with a rapid normalization of the fluorescence of these proteins till the moment of BBB closing.

These findings are consistent with data of others showing that PDT has a direct effect on increasing the gaps of the TJs between endothelial cells via changes of cytoskeleton and vascular tone loss due to microtubule depolymerization [37-39]. One of the mechanisms by which PDT opens the BBB can be an increase in the singlet oxygen/ROS production and development of oxidative stress that we observed in our previous work using 5-ALA [14] and that was also described for phthalocyanines [35]. We assume that a PDT-induced increase in oxidative stress and associated with these changes in TJ proteins might be one mechanism underlying PDT-related BBB opening.

\section{Conclusions}

Our pre-clinical results open a new application of PDT for drug brain delivery. The study of reversible PDT-opening of the BBB using commercial PS such as 5-ALA and AlPcS as well as newly synthesized ZnPc and GalZnPc reveal a stronger PDT-GalZnPc increase in the BBB permeability to EBAC and FITCD vs. other tested PS. These data suggest that PDT-GalZnPc may prove to be a more promising tool for the BBB opening and for drug brain delivery. The limitation of this work is the study of PDT-opening of the BBB in healthy mice. We expect that PDT-opening of the BBB might be a useful tool in the theranostics of gliomas that we will show in our further research. However, these findings represent a first important informative platform that may help the development of novel strategies in the therapy of brain diseases, via optimization of PDT protocol in the light of new insights of PDT effects on the BBB permeability.

Author Contributions: Preparatory work with animals in vivo and ex vivo tissue samples preparation, A.K., I.B. and I.A. (Ilana Agranovich); phthalocyanines synthesis, V.M. and I.E.; novel phthalocyanines photophysical properties evaluation, E.B. and I.A. (Ivan Angelov); spectrofluorimetric analysis A.S., A.T. and M.K.; formal analysis and methodology, A.M., immunohistochemical assay, A.T., A.S., and N.L.; fluorescence spectral measurements and analysis, E.B.; image processing and analysis, E.B. and N.L.; analysis of general data, E.B., J.K. and O.S.-G.; project administration, O.S.-G.; supervision and funding acquisition, E.B. and O.S.-G.

Funding: This research was supported by grant from the Russian Science Foundation, 17-15-01263 (The study of PDT-opening of the BBB), grant 19-15-00201 (The spectrofluorimetric assay of BBB permeability), and grant from the Ministry of High Education and Science, 17.1223.2017/PCh (Synthesis of Ptcs). Spectroscopic studies were supported by grant from the National Science Fund of Bulgaria, \#KP06-N23/8/18.12.18: "Innovative photodynamic methods for treatment of stem cells cultivated from glioblastoma tumours."

Conflicts of Interest: The authors declare that the research was conducted in the absence of any commercial or financial relationships that could be construed as a potential conflict of interest.

\section{References}

1. Bors, L.; Tóth, K.; Tóth, E.; Bajza, Á.; Csorba, A.; Szigeti, K.; Máthé, D.; Perlaki, G.; Orsi, G.; Tóth, G.; et al. Overcoming the Blood-Brain Barrier. Challenges and Tricks for CNS Drug Delivery. Sci. Pharm. 2019, 87, 6. [CrossRef] 
2. Wu, S.; Li, K.; Yan, Y.; Gran, B.; Han, Y.; Zhou, F.; Guan, Y.; Rostami, A.; Zhang, G. Intranasal Delivery of Neural Stem Cells: A CNS-specific, Non-invasive Cell-based Therapy for Experimental Autoimmune Encephalomyelitis. J. Clin. Cell. Immunol. 2013, 4. [CrossRef] [PubMed]

3. Lipsman, N.; Meng, Y.; Bethune, A.; Huang, Y.; Lam, B.; Masellis, M.; Herrmann, N.; Heyn, C.; Aubert, I.; Boutet, A.; et al. Blood-brain barrier opening in Alzheimer's disease using MR-guided focused ultrasound. Nat. Commun. 2018, 9, 2336. [CrossRef]

4. Kiviniemi, V.; Korhonen, V.; Kortelainen, J.; Rytky, J.S.; Keinänen, T.; Tuovinen, T.; Isokangas, M.; Sonkajärvi, E.; Siniluoto, T.; Nikkinen, J.; et al. Real-time monitoring of human blood-brain barrier disruption. PLoS ONE 2017, 12, e0174072. [CrossRef] [PubMed]

5. Gill, S.; Patel, N.; Hotton, G.; O’Sullivan, K.; McCarter, R.; Bunnage, M.; Brooks, D.; Svendsen, C.; Heywood, P. Direct brain infusion of glial cell line-derived neurotrophic factor in Parkinson disease. Nat. Med. 2003, 9, 589-595. [CrossRef]

6. Abdurashitov, A.; Tuchin, V.; Semyachkina-Glushkovskaya, O. Photodynamic therapy of brain tumors and novel optical coherence tomography strategies for in vivo monitoring of cerebral fluid dynamics. J. Innov. Opt. Health Sci. 2019, in press. [CrossRef]

7. Stummer, W.; Stepp, H.; Wiestler, O.D.; Pichlmeier, U. Randomized, Prospective Double-Blinded Study Comparing 3 Different Doses of 5-Aminolevulinic Acid for Fluorescence-Guided Resections of Malignant Gliomas. Neurosurgery 2017, 81, 230-239. [CrossRef]

8. Hirschberg, H.; Berg, K.; Peng, Q. Photodynamic therapy mediated immune therapy of brain tumors. Neuroimmunol. Neuroinflamm. 2018, 5, 27. [CrossRef]

9. Hirschberg, H.; Uzal, F.A.; Chighvinadze, D.; Zhang, M.J.; Peng, Q.; Madsen, M.J. Disruption of the Blood Brain Barrier Following ALA-Mediated Photodynamic Therapy. Lasers Surg. Med. 2008, 40, 535-542. [CrossRef]

10. Madsen, S.J.; Gach, H.M.; Hong, S.J.; Uzal, F.A.; Peng, Q.; Hirschberg, H. Increased nanoparticle-loaded exogenous macrophage migration into the brain following PDT-induced blood-brain barrier disruption. Lasers Surg. Med. 2013, 45, 524-532. [CrossRef]

11. Semyachkina-Glushkovskaya, O.; Kurths, J.; Borisova, E.; Sokolovsky, S.; Mantareva, N.; Angelov, I.; Shirokov, A.; Navolokin, N.; Shushunova, N.; Khorovodov, A.; et al. Photodynamic opening of blood-brain barrier. Biomed. Opt. Exp. 2017, 8, 5040-5048. [CrossRef] [PubMed]

12. Zhang, C.; Feng, W.; Li, Y.; Kurths, J.; Yu, T.; Semyachkina-Glushkovskaya, O.; Zhu, D. Age differences in photodynamic opening of blood-brain barrier through optical clearing skull window in mice. Lasers Surg. Med. 2019, 51, 625-633. [CrossRef] [PubMed]

13. Semyachkina-Glushkovskaya, O.; Chehonin, V.; Borisova, E.; Fedosov, I.; Namykin, A.; Abdurashitov, A.; Shirokov, A.; Khlebtsov, B.; Lyubun, Y.; Navolokin, N.; et al. Photodynamic opening of the blood-brain barrier and pathways of brain clearing pathways. J. Biophotonics 2018, 11, e201700287. [CrossRef] [PubMed]

14. Zhang, C.; Feng, W.; Vodovozova, E.; Tretiakova, D.; Boldyrevd, I.; Li, Y.; Kürths, J.; Yu, T.; Semyachkina-Glushkovskaya, O.; Zhu, D. Photodynamic opening of the blood-brain barrier to high weight molecules and liposomes through an optical clearing skull window. Biomed. Opt. Express 2018, 9, 4850-4862. [CrossRef]

15. Bors, L.; Tóth, K.; Tóth, E.; Bajza, Á.; Csorba, A.; Szigeti, K.; Máthé, D.; Perlaki, G.; Orsi, G.; Tóth, G.; et al. Age-dependent changes at the blood-brain barrier. A Comparative structural and functional study in young adult and middle aged rats. Brain Res. Bull. 2018, 139, 269-277. [CrossRef]

16. Ruk, I.; Pouckova, P.; Benes, J.; Vetrviska, D. Drug Delivery Systems for Phthalocyanines for Photodynamic Therapy. Anticancer Res. 2019, 39, 3323-3339. [CrossRef]

17. Sansaloni-Pastor, S.; Bouilloux, J.; Lange, N. The Dark Side: Photosensitizer Prodrugs. Pharmaceuticals 2019, 12, 148. [CrossRef]

18. Braun, A.; Tcherniac, J. Über die produkte der einwirkung von acetanhy- did auf phthalamid. Ber. Deut. Chem. Ges. 1907, 40, 2709-2714. [CrossRef]

19. Leznoff, C.; Lever, A. Phthalocyanines: Properties and Applications; VCH: Vancouver, BC, Canada, 1989; Volume 2.

20. Lagorio, M.G.; Dicelio, L.E.; San Roman, E.J. Visible and near-IR spectroscopic and photochemical characterization of substituted metallophthalocyanines. Photochem. Photobiol. A Chem. 1993, 72, 153-161. [CrossRef] 
21. Mantareva, V.; Angelov, I.; Wöhrle, D.; Borisova, E.; Kussovski, V. Metallophthalocyanines for antimicrobial photodynamic therapy: an overview of our experience. J. Porphyrins Phthalocyanines 2013, 17, $399-416$. [CrossRef]

22. Xia, P.; Inoguchi, T.; Kern, T.; Engerman, R.; Oates, P.; King, G. Characterization of the mechanism for the chronic activation of diacylglycerol-protein kinase $C$ pathway in diabetes and hypergalactosemia. Diabetes 1994, 43, 1122-1129. [CrossRef] [PubMed]

23. Hempel, A.; Maasch, C.; Heintze, U.; Lindschau, C.; Dietz, R.; Luft, F.; Haller, H. High Glucose Concentrations Increase Endothelial Cell Permeability via Activation of Protein Kinase C $\alpha$. Circ. Res. 1997, 81, 363-371. [CrossRef] [PubMed]

24. Mantareva, V.; Kril, A.; Dimitrov, R.; Wohrle, D.; Angelov, I. Selective photodynamic therapy induced by preirradiation of galactopyranosyl Zn(II) phthalocyanines with UV and red lights. J. Porphyrins Phthalocyanines 2013, 17, 529-539. [CrossRef]

25. Angelov, I.; Kril, A.; Dimitrov, R.; Borisova, E.; Avramov, L.; Mantareva, V. Light enhancement of in vitro antitumor activity of galactosylated phthalocyanines. Photon. Lasers Med. 2016, 5, 123-140. [CrossRef]

26. Mantareva, V.; Petrova, D.; Avramov, L.; Angelov, I.; Borisova, E.; Peeva, M.; Woehrle, D. Long wavelength absorbing cationic Zn (II)-phthalocyanines as fluorescent contrast agents for B16 pigmented melanoma. J. Porphyr. Phthalocyan. 2005, 9, 47-53. [CrossRef]

27. Mantareva, V.; Kril, A.; Angelov, I.; Dimitrov, R.; Borisova, E.; Avramov, L. Effects of the position of galactose units to $\mathrm{Zn}$ (II) phthalocyanine on the uptake and photodynamic activity towards breast cancer cells. Proc. SPIE Biophotonics Photonic Solut. Better Health Care III 2012, 8427, 8427-43.

28. Wang, H.L.; Lai, T.W. Optimization of Evans blue quantitation in limited rat tissue samples. Sci. Rep. 2014, 4, 6588. [CrossRef]

29. Saunders, N.; Dziegielewska, K.; Møllgård, K.; Habgood, M. Markers for blood-brain barrier integrity: how appropriate is Evans blue in the twenty-first century and what are the alternatives? Front. Neurosci. 2015, 9, 385. [CrossRef]

30. Hoffmann, A.; Bredno, J.; Wendland, M.; Derugin, N.; Ohara, P.; Wintermark, M. High and low molecular weight fluorescein isothiocyanate (FITC)-dextran to assess blood-brain barrier disruption: technical consideration. Transl. Stroke Res. 2011, 2, 106-111. [CrossRef]

31. Ribeiro, A.; Tome, J.; Neves, M.; Tome, A.; Cavaleiro, J.; Iamamoto, Y.; Torres, T. [1,2,3,4-Tetrakis( $\alpha / \beta$-D-galactopyranos-6-yl)phthalocyaninato]zinc(II): a water-soluble phthalocyanine. Tetrahedron Lett. 2006, 47, 9177-9180. [CrossRef]

32. Alvares-Mico, X.; Calvete, M.; Hanack, M.; Ziegler, T. Expeditious synthesis of glycosylated phthalocyanines. Synthesis 2007, 14, 2186-2192. [CrossRef]

33. Yamashita, T.; Mimura, K.; Umeda, F.; Kobayashi, K.; Hashimoto, T.; Nawata, H. Increased transendothelial permeation of albumin by high glucose concentration. Metabolism 1995, 44, 739-744. [CrossRef]

34. Chang, K.; Kilo, C.; Williamson, J. Galactose ingestion increases vascular permeability and collagen solubility in normal male rats. J. Clin. Investig. 1987, 79, 367-373. [CrossRef] [PubMed]

35. Ishii, K. Functional singlet oxygen generators based on phthalocyanines. Coord. Chem. Rev. 2012, 256, 1556-1568. [CrossRef]

36. Rick, K.; Sroka, R.; Stepp, H.; Kriegmair, M.; Huber, R.; Jacob, K.; Baumgather, R. Pharmacokinetics of 5aminolevulinic acid-induced protoporphyrin IX in skin and blood. J. Photochem. Photobiol. B Biol. 1997, 40, 313-319. [CrossRef]

37. Hu, S.; Cheng, H.; Zheng, Y.; Zhang, Y.; Yue, W.; Zhang, H. Effects of photodynamic therapy on the ultrastructure of glioma cells. Biomed. Environ. Sci. 2007, 20, 269-273. [PubMed]

38. Sporn, L.; Foster, T. Photofrin and light induces microtubule depolymerization in cultured human endothelial cells. Cancer Res. 1992, 52, 3443-3448.

39. Fingar, V. Vascular effects of photodynamic therapy. J. Clin. Laser Med. Surg. 1996, 14, 323-328. [CrossRef]

(C) 2019 by the authors. Licensee MDPI, Basel, Switzerland. This article is an open access article distributed under the terms and conditions of the Creative Commons Attribution (CC BY) license (http://creativecommons.org/licenses/by/4.0/). 